\title{
Comparison of the effect of electromyogram activity during emergence on anesthetic depth monitoring between phase lag entropy and bispectral index: a prospective observational study
}

\author{
Tae Kyun Kim ${ }^{1,2} \wedge$, Sang-Wook Shin ${ }^{1,2} \wedge$, Hye-Jin Kim ${ }^{1 \wedge}$, Eun-Ji Choi ${ }^{1 \wedge}$, Seyeon Park ${ }^{1}$, So Hyun Kim ${ }^{1}$, \\ Yeong Min Yoo $^{1 \wedge}$, Hee Young $\operatorname{Kim}^{1,2} \wedge$ \\ ${ }^{1}$ Department of Anesthesia and Pain Medicine, Pusan National University Yangsan Hospital, Yangsan, Republic of Korea; ${ }^{2}$ Department of Anesthesia \\ and Pain Medicine, School of Medicine, Pusan National University, Yangsan, Republic of Korea \\ Contributions: (I) Conception and design: TK Kim; (II) Administrative support: TK Kim, HY Kim; (III) Provision of study materials or patients: TK \\ Kim, HY Kim; (IV) Collection and assembly of data: TK Kim, SH Kim, HY Kim; (V) Data analysis and interpretation: TK Kim, HY Kim; (VI) \\ Manuscript writing: All authors; (VII) Final approval of manuscript: All authors. \\ Correspondence to: Hee Young Kim, MD, PhD. Department of Anesthesia and Pain Medicine, School of Medicine, Pusan National University, 20, \\ Geumo-ro, Beomeo-ri, Mulgeumeup, 50612, Yangsan, Republic of Korea. Email: anekhy@gmail.com.
}

\begin{abstract}
Background: The bispectral index (BIS) is the most widely used algorithm for measuring anesthetic depth. The BIS has been demonstrated as inaccurate when neuromuscular blocking drugs (NMBDs) are used. Compared with BIS, phase lag entropy (PLE), which measures the anesthetic depth based on a 4-channel EEG signal, is less affected by EMG. The purpose of this study was to compare the effect of EMG activity during emergence on anesthetic depth monitoring between PLE and BIS.

Methods: Twenty five consecutive patients with physical status I-II of American Society of Anesthesiologists undergoing general anesthesia (age range, 20-60 years). The anesthesiologist attached the sensors of BIS and PLEM 100 on the patient's forehead. NMB reversal was performed by intravenously injecting sugammadex after confirmation of shallow NMB (TOF count 1-4) under neuromuscular monitoring. The BIS and PLE scores were recorded with neuromuscular monitoring at 1-min intervals for 5 min after administration of sugammadex.

Results: The BIS and BIS-EMG measured at 1 min after sugammadex injection were significantly higher at $1 \mathrm{~min}[51.650(46.100,62.225)(\mathrm{P}<0.001) ; 28.500(27.800,31.075)(\mathrm{P}=0.003)]$ than at 0 min. However, there was no between-time point difference in the PLE score and PLE-EMG ( $\mathrm{P}=0.0843, \mathrm{P}=0.329)$.

Conclusions: In general anesthesia using propofol-remifentanil, the BIS at 1 min after sugammadex reversal during emergence appears to be more affected by EMG activity than the PLE score. Therefore, immediately after sugammadex administration (within $1 \mathrm{~min}$ ), it may be clinically useful to evaluate the consciousness status through the PLE score.
\end{abstract}

Keywords: Anesthetic depth; bispectral index (BIS); electroencephalography; emergence; general anesthesia; phase lag entropy (PLE)

Submitted Apr 09, 2021. Accepted for publication Jun 09, 2021.

doi: $10.21037 /$ apm-21-847

View this article at: https://dx.doi.org/10.21037/apm-21-847

\footnotetext{
^ ORCID: Tae Kyun Kim, 0000-0002-4790-896X; Sang-Wook Shin, 0000-0003-1355-7695; Hye-Jin Kim, 0000-0003-1630-0422; Eun-Ji Choi, 0000-0003-3731-0785; Seyeon Park, 0000-0001-7183-1811; So Hyun Kim, 0000-0003-3201-7491; Yeong Min Yoo, 0000-0003-3536-0447; Hee Young Kim, 0000-0001-7809-8739.
} 


\section{Introduction}

There is a need to monitor the level of consciousness (LOC) since intra-anesthetic awareness is a serious mental sequela that can result in post-operative sleep disturbances, nightmares, and anxiety $(1,2)$. The bispectral index (BIS; Aspect Medical System, Inc., Norwood, MA, USA) is the most widely used algorithm in clinical practice for measuring anesthetic depth, which is quantified by bispectral analysis of the different frequency components obtained using a single-channel electroencephalograph (EEG) signal (3). High-frequency signals, including electrical devices, electrocardiograms, electromyograms (EMGs), and epileptiform patterns EEG can increase the BIS, and mislead to use toxic concentration of anesthetics $(4,5)$. Specifically, the BIS has been demonstrated as inaccurate when neuromuscular blocking drugs (NMBDs) are used $(6,7)$. Moreover, the change in the BIS is proportional to the anesthetic agent concentration in patients with complete neuromuscular blockade (NMB) (8-10). However, the BIS may not accurately reflect changes in the anesthetic depth in patients with incomplete NMB or during anesthesia arousal, where NMB reversal occurs. Compared with BIS, phase lag entropy (PLE), which measures the anesthetic depth based on a 4-channel EEG signal, is less affected by EMG (11) (Figure 1).

This study aimed to compare the effect of EMG activity during emergence on anesthetic depth monitoring between PLE and BIS. We present the following article in accordance with the STROBE reporting checklist (available at https:// apm.amegroups.com/article/view/10.21037/apm-21-847/rc).

\section{Methods}

This study was approved by the Institutional Review Board of Pusan National University Yangsan Hospital (Ref: 03-2017-009) and registered on ClinicalTrials. gov (NCT03230929). This study included voluntary participants and written informed consent was obtained before the study was conducted. The exclusion criteria were lesions that can affect EEG, including cerebral hemorrhage, cerebral apoplexy, and epilepsy, and diseases that can affect EMG, including muscular dystrophy, muscle stiffness, inflammatory muscular lesions, metabolic myopathy, congenital myopathy, and myasthenia gravis. The study was conducted in accordance with the Declaration of Helsinki (as revised in 2013).

This study included 25 consecutive patients with

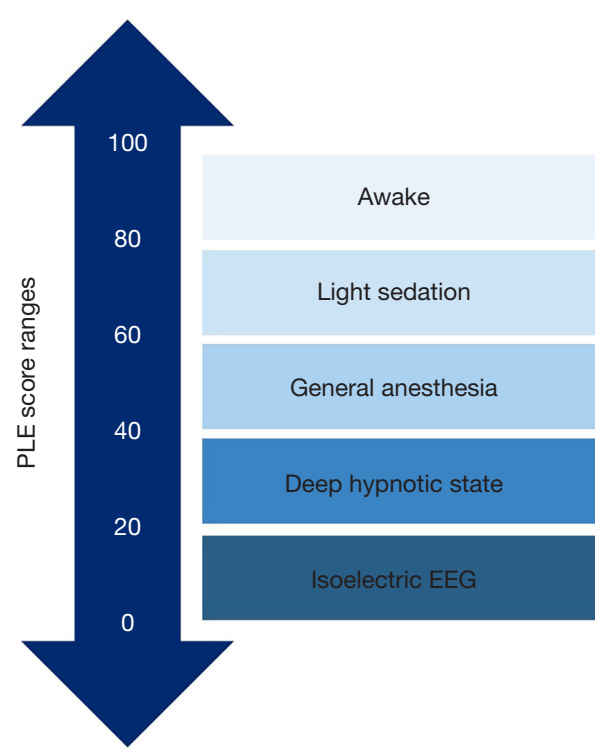

Figure 1 PLE score range guideline. PLE, phase lag entropy; EEG, electroencephalograph.

physical status I-II of American Society of Anesthesiologists undergoing general anesthesia (age range, 20-60 years) from August 11, 2017, to November 10, 2017. The anesthesiologist attached the sensors of BIS and PLEM 100 (Inbody Co., Ltd, Seoul, Korea) on the patient's forehead; moreover, a neuromuscular monitoring device (Philips IntelliVue NMT Module 865,383, Phillips Healthcare, Amsterdam, the Netherlands) was attached to the medial wrist side and ipsilateral thumb for continuous monitoring of the state of consciousness and NMB before, during, and after surgery. For total intravenous anesthesia, 2\% propofol and remifentanil were intravenously administered using a target-controlled infusion pump. After intravenous injection of rocuronium $0.6 \mathrm{mg} / \mathrm{kg}$ for $\mathrm{NMB}$, endotracheal intubation was performed. The degree of NMB was intraoperatively maintained within the deep block [trainof-four (TOF) count $<2$ ]; moreover, the $2 \%$ propofol concentration was adjusted to maintain the BIS and PLE scores between 40 and 60 during operation. There was no change of patients' position during emergence. $\mathrm{NMB}$ reversal was performed by intravenously injecting $2 \mathrm{mg} / \mathrm{kg}$ sugammadex after confirmation of shallow NMB (TOF count 1-4) under neuromuscular monitoring while infusion of the $2 \%$ propofol and remifentanil was stopped after confirmation of the BIS and PLE scores between 40 and 60 . Subsequently, the BIS and PLE scores were recorded with neuromuscular monitoring at 1 -min intervals for $5 \mathrm{~min}$. 


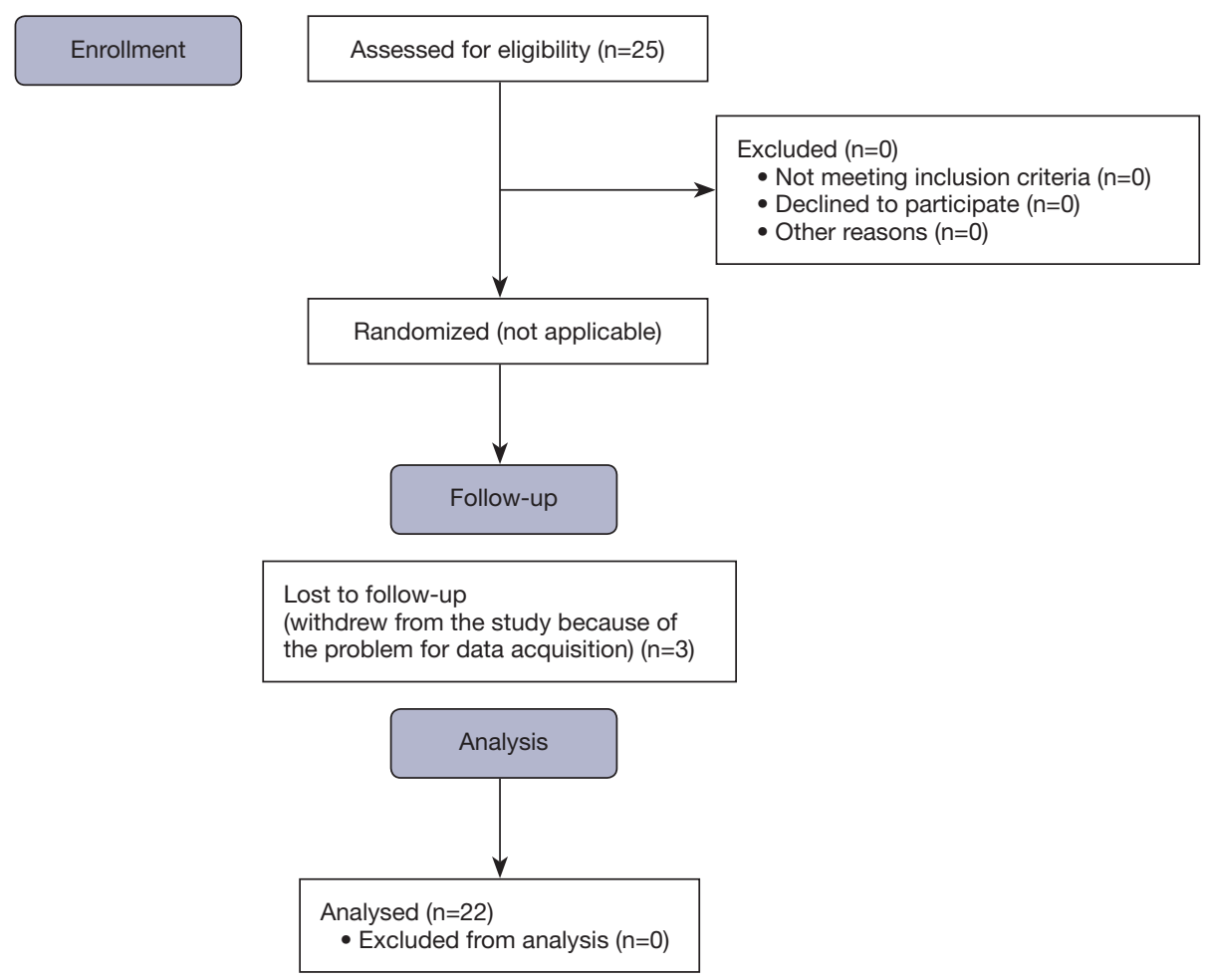

Figure 2 Consort flow diagram.

Table 1 Patients characteristics

\begin{tabular}{lc}
\hline Variable & $\begin{array}{c}\mathrm{N}(\%) \text { or } \\
\text { mean } \pm \mathrm{SD}\end{array}$ \\
\hline Gender & $8(36.4)$ \\
Male & $14(63.6)$ \\
Female & $45.17 \pm 9.83$ \\
Age (yrs) & $168.46 \pm 7.63$ \\
Height (cm) & $70.98 \pm 16.42$ \\
Weight (kg) & \\
ASA class & $12(54.5)$ \\
1 & $10(45.5)$ \\
2 & $62.27 \pm 13.05$ \\
Duration of operation (min) & $68.77 \pm 29.48$ \\
Total amount of rocuronium (mg) & \\
TOF & \\
Count at the immediate end of operation & $0.80 \pm 0.32$ \\
Ratio 5 min after administration of sugammadex (\%) & $97.80 \pm 11.92$ \\
\hline Normal continuous variables was expressed as mean \pm SD. SD, \\
standard deviation; ASA, American Society of Anesthesiologists; \\
TOF, train-of-four.
\end{tabular}

After NMB recovery, the trachea was extubated and the patient was transferred to the post-anesthesia care unit.

\section{Statistical analysis}

We required a sample size of 20 to achieve $80 \%$ power for detecting a mean of paired differences of 8.3 , with an estimated standard deviation of differences of 12.5 and a significance level (alpha) of 0.05 , using a two-sided paired $t$-test with reference to Seo et al.'s study (12). Student's $t$-test was used to analyze the differences in the BIS and PLE scores after sugammadex administration. Data were expressed as median (Q1, quartile 1-Q3, quartile 3) or mean \pm standard deviation. Statistical significance was set at $\mathrm{P}<0.05$. All statistical analyses were performed using IBM SPSS Statistics 25.0 (IBM Corp., Armonk, NY, USA).

\section{Results}

We recruited 25 patients; among them, we excluded three patients with data acquisition problems (Figure 2). Table 1 presents the demographic data of the participants. The BIS and BIS-EMG measured at $1 \mathrm{~min}$ after sugammadex 
Table 2 BIS and PLE values at 0 and 1 minute after administration of sugammadex $(\mathrm{N}=22)$

\begin{tabular}{lccc}
\hline Value & 0 min & 1 min & P value \\
\hline BIS & $49.650(43.575,58.125)$ & $51.650(46.100,62.225)$ & $<0.001$ \\
BIS-EMG $(\mathrm{dB})$ & $28.050(27.400,30.000)$ & $28.500(27.800,31.075)$ & 0.003 \\
PLE & $52.545 \pm 8.163$ & $53.727 \pm 9.130$ & 0.0843 \\
PLE-EMG (dB) & $18.000(15.000,21.750)$ & $17.500(13.000,26.250)$ & 0.329 \\
\hline
\end{tabular}

Non-normal continuous variables was expressed as median (Q1-Q3) and normal continuous variables as mean \pm SD. BIS, bispectral index; PLE, phase lag entropy; EMG, electromyography; SD, standard deviation; Q1, quartile 1; Q3, quartile 3.

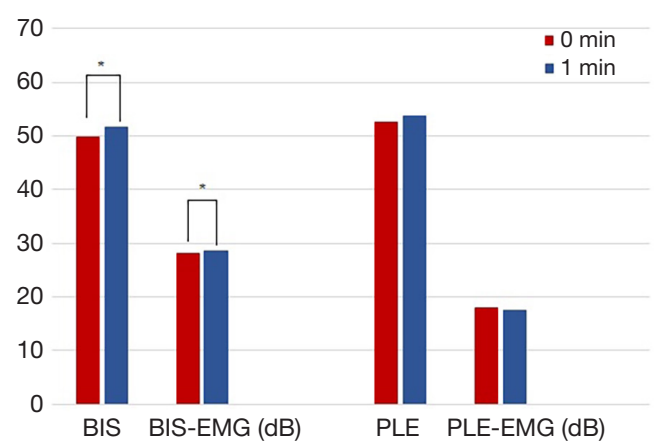

Figure 3 BIS and PLE values at 0 and 1 minute after administration of sugammadex $(\mathrm{N}=22)$. * $\mathrm{P}<0.05$. BIS, bispectral index; EMG, electromyography; PLE, phase lag entropy.

injection were significantly higher $[51.650 \mathrm{~dB}(46.100$, $62.225 \mathrm{~dB})(\mathrm{P}<0.001) ; 28.500 \mathrm{~dB}(27.800,31.075 \mathrm{~dB})$ $(\mathrm{P}=0.003)]$ than at $0 \mathrm{~min}$. However, there was no betweentime point difference in the PLE score and PLE-EMG $(\mathrm{P}=0.0843, \mathrm{P}=0.329)$ (Table 2, Figure 3).

According to study of Schuller et al. (13), the BIS-EMG value of 35 was used as a cutoff value, and when the BISEMG was $<35$ after 5 min of sugammadex administration, there was no difference in the BIS score at 0 and $5 \mathrm{~min}$. However, when the BIS-EMG was $>35$ after $5 \mathrm{~min}$, there was a significant difference in the BIS score after $5 \mathrm{~min}$ (69.300 \pm 9.584$)$ and $0 \min (55.108 \pm 10.472)(\mathrm{P}=0.001)$. There was no difference in the PLE score after 0 and 5 min even when the PLE-EMG was $<35$ after $5 \mathrm{~min}$. On the other hand, when the PLE-EMG was $\geq 35$ after $5 \mathrm{~min}$, there was a significant difference in the PLE score after $5 \mathrm{~min}$ $(67.846 \pm 12.773)$ and $0 \min (54.769 \pm 7.780)(\mathrm{P}=0.004)$ (Table 3, Figure 4).

\section{Discussion}

This study compared the BIS and PLE scores during emergence in patients who received propofol-remifentanil anesthesia. In this study, the BIS and BIS-EMG measured at 0 and $1 \mathrm{~min}$ after sugammadex injection were all high at $1 \mathrm{~min}$ and showed significant differences. However, there was no difference in the PLE score and PLE-EMG between 0 and $1 \mathrm{~min}$. NMB recovery can be assumed to have occurred by 1 min after sugammadex reversal, with an increase in the BIS-EMG and BIS. This is consistent with a previous report that $\mathrm{NMB}$ recovery can be restored to a $\mathrm{T} 4 / \mathrm{T} 1$ ratio of 0.9 at approximately $1 \mathrm{~min}$ after sugammadex administration in a shallow NMB (TOF count 1-4) (14). Contrastingly, there was no significant difference in the PLE-EMG at 1 min after sugammadex administration. BIS-EMG is a logarithmic scale of the total signal power between 70 and $110 \mathrm{~Hz}$ while PLE-EMG is a calculated parameter using signals $>45 \mathrm{~Hz}$ (12). Additionally, when the EMG was $<35$ after 5 min of sugammadex reversal, there was no difference in both the BIS and PLE score after 0 and $5 \mathrm{~min}$. However, when the EMG score was $\geq 35$ after 5 min, the BIS and PLE scores after 5 min were significantly greater than those after 0 min. After 5 min of sugammadex reversal, consciousness recovery has already progressed; moreover, similar to the $\mathrm{BIS}$, the PLE score at $5 \mathrm{~min}$ increases compared with that at 0 min when the EMG was $\geq 35$.

LOC evaluation during general anesthesia is important for preventing intra-anesthetic arousal and excessive administration of anesthetic agents, with the use of BIS being clinically useful (15). The LOC is associated with the complex and diverse linkages between brain regions (16), which are reduced during general anesthesia (17). Emergence from general anesthesia is a vital process; moreover, compared with using clinical signs such as eye opening, extubation, and orientation, using parameters including the BIS improves the quality of recovery (18). It is important to control concentration of anesthetics, and the level of anesthesia delicately under the guidance of the BIS, which is one of the most widely used processed EEG-based 
Table 3 BIS and PLE values at 0 and 5 minutes according to EMG value at 5 minutes, after administration of sugammadex

\begin{tabular}{lcc}
\hline Value & 0 min & 5 min \\
\hline BIS with EMG_5 $\min <35 \mathrm{~dB}(\mathrm{~N}=9)$ & $46.489 \pm 8.042$ & $53.833 \pm 7.116$ \\
BIS with EMG_5 $\min \geq 35 \mathrm{~dB}(\mathrm{~N}=13)$ & $55.108 \pm 10.472$ & $69.300 \pm 9.584$ \\
PLE with EMG_5 $\min <35 \mathrm{~dB}(\mathrm{~N}=9)$ & $49.333 \pm 8.031$ & $54.778 \pm 5.333$ \\
PLE with EMG_5 $\min \geq 35 \mathrm{~dB}(\mathrm{~N}=13)$ & $54.769 \pm 7.780$ & $67.846 \pm 12.773$ \\
\hline
\end{tabular}

Normal continuous variables was expressed as mean \pm SD. BIS, bispectral index; PLE, phase lag entropy; EMG, electromyography.

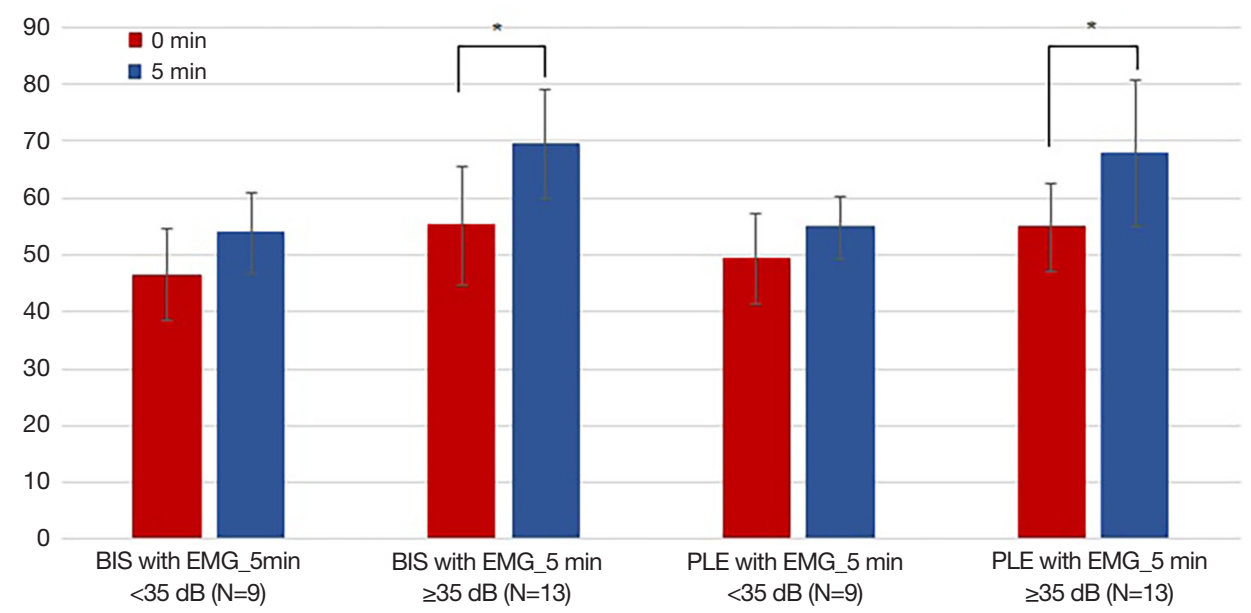

Figure 4 BIS and PLE values at 0 and 5 minutes according to EMG value at 5 minutes, after administration of sugammadex. *, P<0.05. BIS, bispectral index; EMG, electromyography; PLE, phase lag entropy.

parameter for monitoring anesthetic depth $(3,19)$. However, the BIS has limitations because the BIS and BIS-EMG are obtained using EEG signals in the range of $0-47 \mathrm{~Hz}$ and $70-110 \mathrm{~Hz}$, respectively. Since EMG signals exist in the $30-300 \mathrm{~Hz}$ range, those in the $30-47 \mathrm{~Hz}$ range can be interpreted as EEG signals, which can falsely indicate an elevated BIS or low BIS-EMG value (3). Messner et al. reported that BIS simultaneously decreased with EMG activity disappearance after succinylcholine administration in awake patients (6); further, Liu et al. reported that NMBD decreased EMG activity and therefore reduces the BIS (20). Additionally, based on the afferentation theory that the muscle stretch receptor stimulates the brain's arousal center (21), NMBD administration can reduce the BIS by blocking the muscle stretch receptor and reducing the stimulation that causes brain arousal. A previous study reported no changes in the BIS score even after $10 \mathrm{~min}$ of sugammadex reversal (22); however, the EMG activity was a single entity rather than dividing it into low and high groups, which may differ from other studies.
The PLE monitor has been recently approved for clinical use, with several studies assessing its usefulness and comparison with the BIS; moreover, it has been shown as useful for LOC evaluation in general anesthesia $(11,12,23,24)$. PLE employs EEG signals with ranges of $0.1-1$ and $32-45 \mathrm{~Hz}$ while PLE-EMG uses signals $>45 \mathrm{~Hz}$. Therefore, PLE can interpret $30-45 \mathrm{~Hz}$ EMG as an EEG signal; however, PLE obtains a phase relationship from two channels and converts it into entropy after binarized calculation. Additionally, electrical artifacts may occur on the two EEG channels; however, they are removed during binarization (12). Therefore, theoretically, the PLE score could be less affected by EEG than the BIS; however, there have been few studies on the relationship between the PLE score and PLE-EMG. Although several studies showed PLE score has correlation with BIS for evaluating consciousness and depth of anesthesia during general anesthesia $(11,12,24)$, the present study is meaningful since it is the first to investigate the PLE-EMG effect on the PLE score. Additionally, a previous study reported that the BIS does 
not increase during deep propofol-remifentanil anesthesia (BIS 30-33) and NMB reversal using sugammadex (22); moreover, the BIS in this study was $>45$ after sugammadex reversal $(0,1,5 \mathrm{~min})$, which could be sufficiently influenced and increased by reversal.

This study has several limitations. First, since two sensors were attached to each patient's forehead, there were slightly different sensor attachment sites, which could result in differences in the BIS, BIS-EMG, PLE, and PLEEMG measurements. Second, we did not measure the TOF count or ratio at $1 \mathrm{~min}$ after sugammadex administration. However, sugammadex can reverse deep NMB within 3 min (25) and a shallow NMB to T4/T1 ratio 0.9 after 1 min (14). Third, the surgery type was not unified; therefore, the effect of nociceptive-antinociceptive balance on LOC and EEG could not be excluded. Third, although BIS-EMG measured at 1 min than 0 min after sugammadex injection was significantly higher, the difference of mean value is about $0.5 \mathrm{~dB}$. This can lead to doubts of clinical significance. However, the maximal or normal BIS-EMG value is about $60 \mathrm{~dB}$ by the study of Schuller et al. (13), and tiny change of BIS-EMG value could have clinical significance.

In conclusion, in general anesthesia using propofolremifentanil, the BIS at 1 min after sugammadex reversal during emergence appears to be more affected by EMG activity than the PLE score. Furthermore, after 5 min, there was no difference in the effect of EMG activity on the BIS and PLE score. Therefore, immediately after sugammadex administration (within $1 \mathrm{~min}$ ), it may be clinically useful to evaluate the consciousness status through the PLE score.

\section{Acknowledgments}

Funding: This work was supported by Inbody Co., Ltd., Seoul, Korea.

\section{Footnote}

Reporting Checklist: The authors have completed the STROBE reporting checklist. Available at https://apm. amegroups.com/article/view/10.21037/apm-21-847/rc

Data Sharing Statement: Available at https://apm.amegroups. com/article/view/10.21037/apm-21-847/dss

Conflicts of Interest: All authors have completed the ICMJE uniform disclosure form (available at https://apm. amegroups.com/article/view/10.21037/apm-21-847/coif). TKK reports funding from Inbody Co., Ltd., Seoul, Korea. The other authors have no conflicts of interest to declare.

Ethical Statement: The authors are accountable for all aspects of the work in ensuring that questions related to the accuracy or integrity of any part of the work are appropriately investigated and resolved. This study was approved by the Institutional Review Board of Pusan National University Yangsan Hospital (Ref: 03-2017-009) and registered on ClinicalTrials.gov (NCT03230929). This study included voluntary participants and written informed consent was obtained before the study was conducted. The study was conducted in accordance with the Declaration of Helsinki (as revised in 2013).

Open Access Statement: This is an Open Access article distributed in accordance with the Creative Commons Attribution-NonCommercial-NoDerivs 4.0 International License (CC BY-NC-ND 4.0), which permits the noncommercial replication and distribution of the article with the strict proviso that no changes or edits are made and the original work is properly cited (including links to both the formal publication through the relevant DOI and the license). See: https://creativecommons.org/licenses/by-nc-nd/4.0/.

\section{References}

1. Ghoneim MM. Awareness during anesthesia. Anesthesiology 2000;92:597-602.

2. American Society of Anesthesiologists Task Force on Intraoperative Awareness. Practice advisory for intraoperative awareness and brain function monitoring: a report by the american society of anesthesiologists task force on intraoperative awareness. Anesthesiology 2006;104:847-64.

3. Johansen JW. Update on bispectral index monitoring. Best Pract Res Clin Anaesthesiol 2006;20:81-99.

4. Hemmerling TM, Fortier JD. Falsely increased bispectral index values in a series of patients undergoing cardiac surgery using forced-air-warming therapy of the head. Anesth Analg 2002;95:322-3, table of contents.

5. Stasiowski MJ, Duława A, Król S, et al. Polyspikes and Rhythmic Polyspikes During Volatile Induction of General Anesthesia With Sevoflurane Result in Bispectral Index Variations. Clin EEG Neurosci 2020;1550059420974571.

6. Messner M, Beese U, Romstöck J, et al. The bispectral 
index declines during neuromuscular block in fully awake persons. Anesth Analg 2003;97:488-91, table of contents.

7. Bruhn J, Bouillon TW, Shafer SL. Electromyographic activity falsely elevates the bispectral index. Anesthesiology 2000;92:1485-7.

8. Dahaba AA, Mattweber M, Fuchs A, et al. The effect of different stages of neuromuscular block on the bispectral index and the bispectral index-XP under remifentanil/ propofol anesthesia. Anesth Analg 2004;99:781-7, table of contents.

9. Greif R, Greenwald S, Schweitzer E, et al. Muscle relaxation does not alter hypnotic level during propofol anesthesia. Anesth Analg 2002;94:604-8; table of contents.

10. Vasella FC, Frascarolo P, Spahn DR, et al. Antagonism of neuromuscular blockade but not muscle relaxation affects depth of anaesthesia. Br J Anaesth 2005;94:742-7.

11. Shin HW, Kim HJ, Jang YK, et al. Monitoring of anesthetic depth and EEG band power using phase lag entropy during propofol anesthesia. BMC Anesthesiol 2020;20:49.

12. Seo KH, Kim KM, Lee SK, et al. Comparative Analysis of Phase Lag Entropy and Bispectral Index as Anesthetic Depth Indicators in Patients Undergoing Thyroid Surgery with Nerve Integrity Monitoring. J Korean Med Sci 2019;34:e151.

13. Schuller PJ, Newell S, Strickland PA, et al. Response of bispectral index to neuromuscular block in awake volunteers. Br J Anaesth 2015;115 Suppl 1:i95-i103.

14. Pühringer FK, Gordon M, Demeyer I, et al. Sugammadex rapidly reverses moderate rocuronium- or vecuroniuminduced neuromuscular block during sevoflurane anaesthesia: a dose-response relationship. Br J Anaesth 2010;105:610-9.

15. Ekman A, Lindholm ML, Lennmarken C, et al. Reduction in the incidence of awareness using BIS monitoring. Acta Anaesthesiol Scand 2004;48:20-6.

16. Dehaene S, Changeux JP. Experimental and theoretical approaches to conscious processing. Neuron 2011;70:200-27.

Cite this article as: Kim TK, Shin SW, Kim HJ, Choi EJ, Park S, Kim SH, Yoo YM, Kim HY. Comparison of the effect of electromyogram activity during emergence on anesthetic depth monitoring between phase lag entropy and bispectral index: a prospective observational study. Ann Palliat Med 2022;11(4):12901296. doi: 10.21037/apm-21-847
17. Alkire MT, Hudetz AG, Tononi G. Consciousness and anesthesia. Science 2008;322:876-80.

18. Punjasawadwong $\mathrm{Y}$, Phongchiewboon A, Bunchungmongkol N. Bispectral index for improving anaesthetic delivery and postoperative recovery. Cochrane Database Syst Rev 2014;2014:CD003843.

19. Avidan MS, Palanca BJ, Glick D, et al. Protocol for the BAG-RECALL clinical trial: a prospective, multicenter, randomized, controlled trial to determine whether a bispectral index-guided protocol is superior to an anesthesia gas-guided protocol in reducing intraoperative awareness with explicit recall in high risk surgical patients. BMC Anesthesiol 2009;9:8.

20. Liu N, Chazot T, Huybrechts I, et al. The influence of a muscle relaxant bolus on bispectral and datex-ohmeda entropy values during propofol-remifentanil induced loss of consciousness. Anesth Analg 2005;101:1713-8.

21. Lanier WL, Iaizzo PA, Milde JH, et al. The cerebral and systemic effects of movement in response to a noxious stimulus in lightly anesthetized dogs. Possible modulation of cerebral function by muscle afferents. Anesthesiology 1994;80:392-401.

22. Illman H, Antila H, Olkkola KT. Reversal of neuromuscular blockade by sugammadex does not affect EEG derived indices of depth of anesthesia. J Clin Monit Comput 2010;24:371-6.

23. Ki S, Kim KM, Lee YH, et al. Phase lag entropy as a hypnotic depth indicator during propofol sedation. Anaesthesia 2019;74:1033-40.

24. Park JH, Lee SE, Kang E, et al. Effect of depth of anesthesia on the phase lag entropy in patients undergoing general anesthesia by propofol: A STROBE-compliant study. Medicine (Baltimore) 2020;99:e21303.

25. Lee C, Jahr JS, Candiotti KA, et al. Reversal of profound neuromuscular block by sugammadex administered three minutes after rocuronium: a comparison with spontaneous recovery from succinylcholine. Anesthesiology 2009;110:1020-5. 